Note

\title{
How to assess fin damage in rainbow trout, Oncorhynchus mykiss?
}

\author{
Jeannine Person-Le Ruyet ${ }^{\mathrm{a}}$, Nicolas Le Bayon and Sylvie Gros \\ IFREMER Centre de Brest, UMR 1067, BP 70, 29280 Plouzané, France
}

Received 10 April 2007; Accepted 8 June 2007

\begin{abstract}
Fin condition was examined in 100-500 g hatchery-reared rainbow trout sampled in a large range of environmental conditions of an experimental farm. A method that is simple to use, requires little training, and does not need costly sophisticated equipment or reference to a model is proposed to assess fin erosion (defined as a decrease in size and profile alteration of fins). A procedure to reliably measure the length of the longest ray present in two selected fins (dorsal and pectoral) is described. Fin general profile is assessed using five levels of erosion based on the precise rank of intact or eroded rays to limit subjective bias. The suitability of the two descriptors of fin erosion under laboratory, farm or field conditions is discussed.
\end{abstract}

Key words: Rainbow trout / Oncorhynchus mykiss / Fin erosion

\section{Introduction}

Despite differences in fish perception from consumers, farmers and people anxious about ethic and animal protection, there is an increasing interest in fish appearance; fish should look healthy and aesthetically pleasing without visible signs of suffering or malformation. The commercial value of hatcheryreared juveniles is closely related to the lack of skeletal malformation and, for some purposes, also related to fin condition. In stock enhancement programs, fin condition is critical as it may compromise release success, or desirability to sport anglers. There is extensive literature on the causes of fin erosion, especially in Salmonids (Bosakowski and Wagner 1995; Arndt et al. 2002; Pelis and McCormick 2003; Latremouille 2003; St-Hilaire et al. 2006). Fin condition is used as an indicator of hatchery origin, to separate different species or strains in natural environments or to describe phenotypic diversity (Bosakowski and Wagner 1994; Danner and Boucher 2005; Zimmerman et al. 2006). It can provide, along with other organismic indices, a relatively simple and rapid indicator of health condition in farms and in the wild where, except in degraded environments, the occurrence of skin injuries and fin damages (erosion and necrosis) is lower than in aquaculture (Goede and Barton 1990; Latremouille 2003). Fin erosion is also considered as an interesting candidate to assess fish welfare, a recent public concern that refers to the quality of life

\footnotetext{
a Corresponding author: jperson@ifremer.fr
}

or state of well being of fish (Ellis et al. 2002; Turnbull et al. 2005; Huntingford et al. 2006; Ashley 2007).

Fin condition can be described by fin length and profile. Relative fin indexes that are expressed as the ratio of fin length to total fish length are routinely used. These ratios assume that fin length grows in proportion to overall body length, whatever the environmental conditions. They are calculated for a specific fin, selected fins or all rayed fins, and comparisons between the different indexes are difficult especially since there is no standardized method for measuring fin length. For dorsal fin, for example, fin length may be the length of first rays at the fin insertion, the length of the longest ray present, the average length of all rays or the length at the median point of attachment to fish body (Kindschi 1997; Barrow and Lellis 1999; Bosakowski and Wagner 1994; Zimmerman et al. 2006; St-Hilaire et al. 2006). Fin profile damage is also estimated in many ways: the percentage of missing rays, percentage or fraction of intact extremities present in each fin or in all fins, or the percentage of severe or mild active erosion (Goede and Barton 1990; Latremouille 2006). Latremouille (2006) comments that all fin erosion procedures are partly subjective and the degree of erosion is difficult to estimate without reference to a perfect fin, often missing in the population studied. The different indexes used to score the condition of specific or total fins mainly refer to major fin damage (erosion, necrosis, nipping and rot) which compromises fish health and survival, and there is little objective information available relating to 

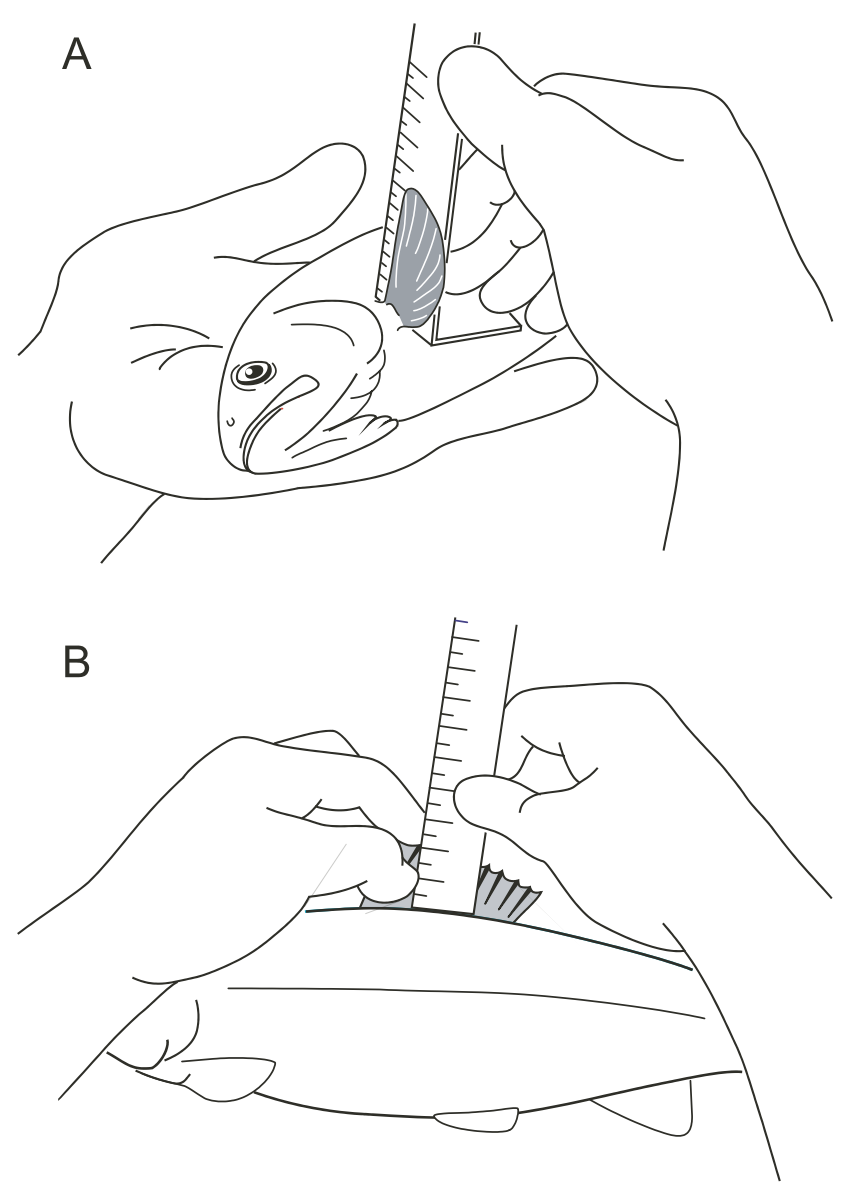

Fig. 1. Procedure for measuring the maximum length of (A) the left pectoral or (B) dorsal fin of $O$. mykiss.

the use of slight fin erosion as a barometer of fish welfare (St-Hilaire et al. 2006; Hoyle et al. 2007). There is a need for a method that can rapidly quantify, in a reliable way, small amounts of erosion of selected fins, preferably to all rayed fins, and that can be easily applied to any species or environmental condition (aquaculture or the natural environment).

The aim of this study was to find the most accurate method, not affected by subjective bias and easy to use for any operator, to rapidly assess fin erosion in rainbow trout (35000 metric tons are produced per year in France under intensive rearing conditions that may compromise fish welfare). Fin erosion is defined as a decrease in size and profile alteration of fins and is determined in two fins, pectoral and dorsal.

\section{Materials and methods}

Fin condition was examined in 100-500 g rainbow trout of an autumnal INRA-strain sampled in a large range of environmental conditions at Semii fresh water facilities (Sizun, Brittany). Fins of fish held in a large tank $\left(50 \mathrm{~m}^{3}\right)$ under the best-known environmental conditions (stocking density $<3 \mathrm{~kg} \mathrm{~m}^{-3}$ ) were used as a reference as they were perfect fins (long fin with intact rays and a reference profile). They were compared to fins of the same initial population reared under usual or extreme experimental conditions that may have led to
A
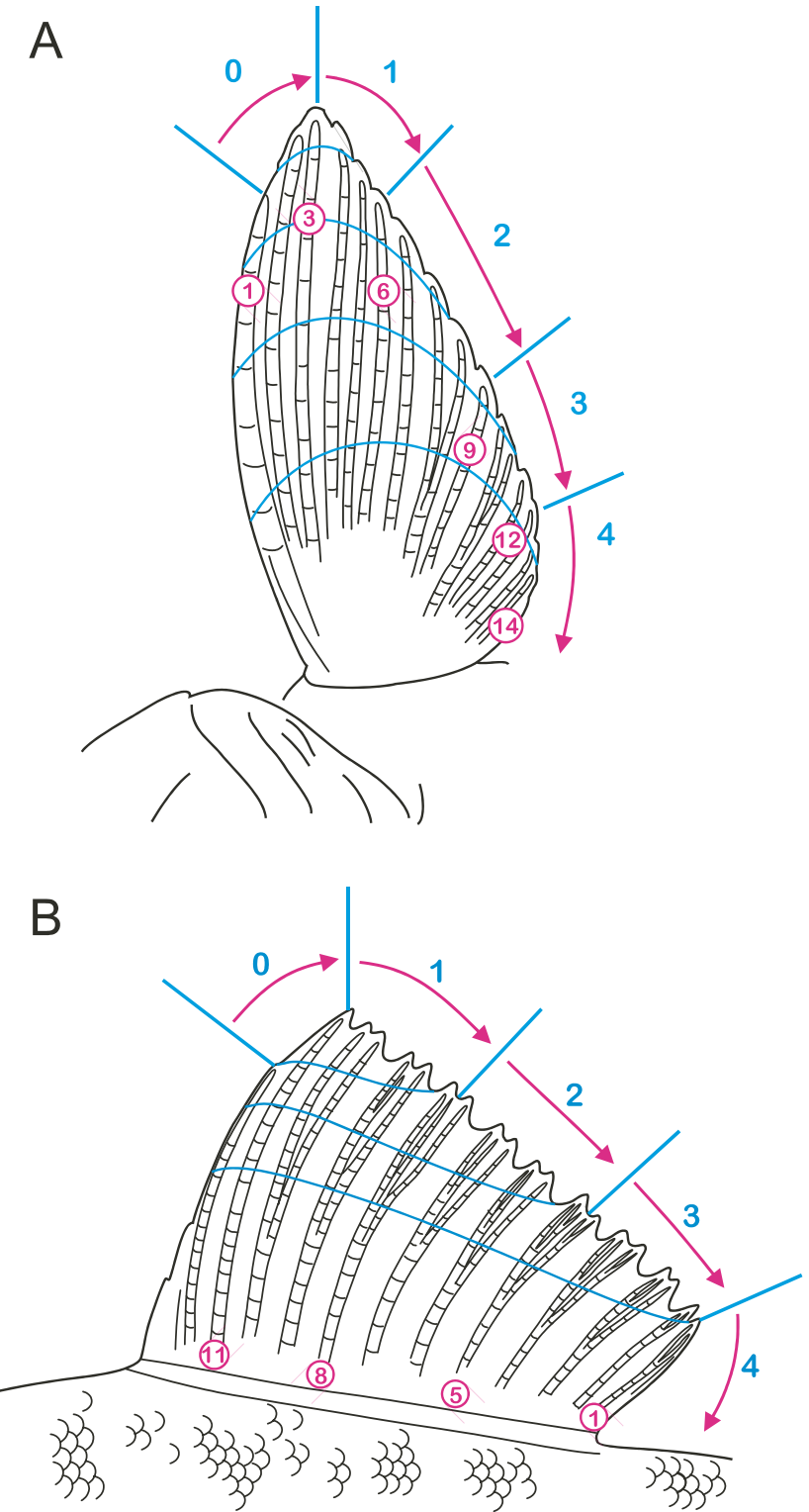

Fig. 2. Diagram of the 5 erosion levels of (A) the left pectoral and (B) dorsal fin of $O$. mykiss based on the rank of intact rays (figures inside a ring), with the related fin profile (red lines).

fin damage caused by interactions with other fish (competition for food and/or space), contact with tank walls (fin abrasion), or handling procedures (regular weighing).

The dorsal and pectoral fins were selected as they were shown to be the most relevant fins to quantify erosion (contrat 98/2-INRA-IFREMER); this finding is in agreement with other studies (Bosakowski and Wagner 1994; St-Hilaire et al. 2006). Several hundred fish were observed to determine erosion processes and kinetic of dorsal and pectoral fins. Fins of 62 anaesthetized fish (ethylene-glycol-monophenyl-ether, $0.5 \%$ ) were initially examined to develop a method, then the method was tested using 39 fish with fin damage (no absent fins observed in the experimental farm). Fish were successively examined by 4 operators, including one naive person, and the time allocated to check the length and level of erosion 
Typical erosion levels of a pectoral fin of Oncorhynchus mykiss (differences in scale between slides)

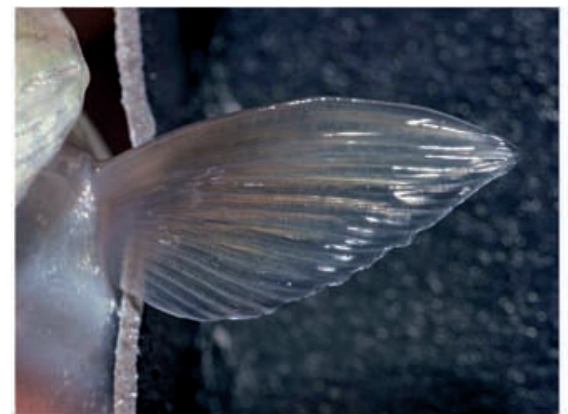

A, level 0

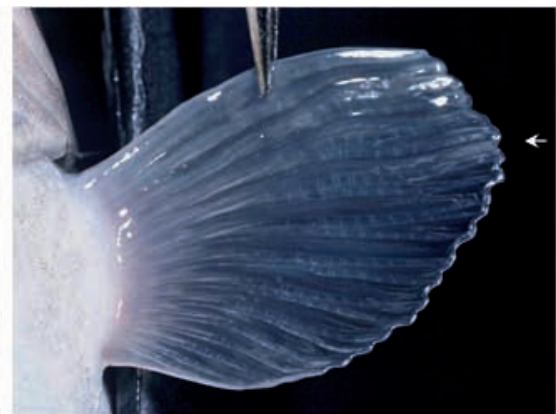

B, level 1

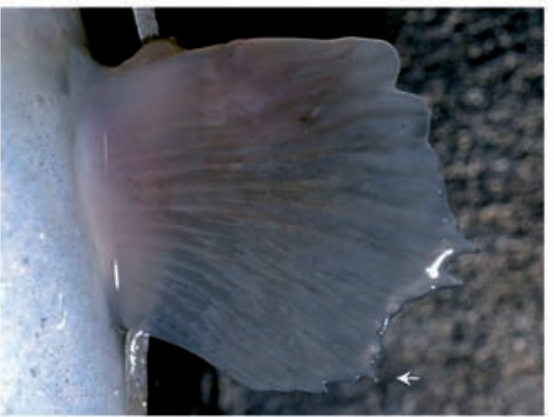

D, level 3

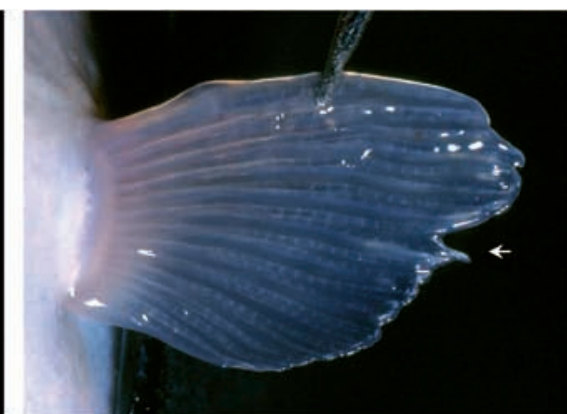

C, level 2

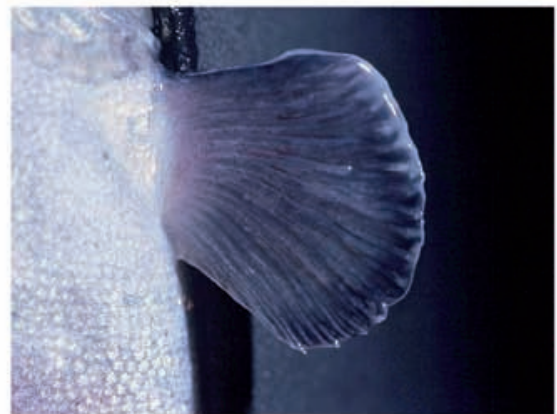

E, level 4

Fig. 3. Typical erosion levels of a pectoral fin of $O$ mykiss. A, level 0; B, level 1; C, level 2, D level 3; E, level 4. The arrow indicates the rank of last intact ray; the slides are not at the same scale.

of the dorsal and the two pectorals was one minute per fish. This first challenge test showed minor deviations only for the naive person and dorsal fin while there was full agreement between the 3 trained operators for all fin erosion levels (Non significant differences, $p<0.05)$. For the naive person there was some confusion about level 1 and 2 in $10 \%$ of these fish groups requiring refinement of erosion levels for the dorsal fin. Two operators, to avoid any possible observer bias, tested a modified procedure on 32 fish simultaneously and fin rays were recorded (direct observation, Figs. 3 and 4). Final procedure was established in 3 successive steps using 133 fish up to full agreement of the operators. An intra-operator test was also conducted using 40 other fish to validate the method reproducibility. This procedure has been finally applied to 2662 fish, autumnal INRA and Mirwart strains, when testing the effects of stocking density on rainbow trout welfare (results will be presented in a coming paper).

\section{Results}

\subsection{Pectoral fin}

In rainbow trout (autumnal INRA strain), the number of rays of each pectoral fin was $14.4 \pm 0.8$ (mean \pm SE, $n=37$ ): one spine and 13-14 soft rays (2-3 rays with a single dichotomy). In a perfect fin, the 3rd ray is the longest. Pectoral fins are normally symmetrical but they can be differently affected by culture conditions so there is a need to check both right and left pectoral fins. Vertical slits are also common, only
Table 1. The 5 levels of erosion of dorsal and pectoral fins.

\begin{tabular}{cll}
\hline Level & \multicolumn{1}{c}{$\begin{array}{c}\text { Pectoral fin } \\
\text { Intact } \text { ray from }\end{array}$} & $\begin{array}{c}\text { Dorsal fin } \\
\text { Intact ray } \text { from }\end{array}$ \\
\hline $\mathbf{0}$ & R1 to R3 included & R12 \\
$\mathbf{1}$ & R4 to R6 included & R11 to R8 \\
$\mathbf{2}$ & R7 to R9 included & R7 to R5 \\
$\mathbf{3}$ & R10 to R12 included & R4 to R1 \\
$\mathbf{4}$ & R13 to R14 & no intact ray \\
& or no intact ray & (R13-14 excluded). \\
\hline
\end{tabular}

major slits are recorded considering that it is divided when one slit length represents at least half the length of the two contiguous rays.

Pectoral highest length is measured as reported in Fig. 1A. In a fin with one or many slits, the length is taken at the longest lobe (measuring when necessary, the length of each lobe).

A scale of erosion with 5 levels is used to characterize pectoral profile (Table 1). As reported in Fig. 2A, each degree of erosion refers to the rank of the last intact ray (first ray, R1, on dorsal side).

\subsection{Dorsal fin}

The number of rays of the dorsal fin was $14.2 \pm 0.4$ (mean \pm SE, $n=37$ ): 3-4 spines and 10-11 soft rays with many dichotomies in some rays. In a perfect dorsal fin the two longest rays are the 4th and 5th rays (the first two soft rays following the spines). The presence of vertical slits is noticed as for pectoral fins. 
Typical erosion levels of a dorsal fin of Oncorhynchus mykiss (differences in scale between slides)

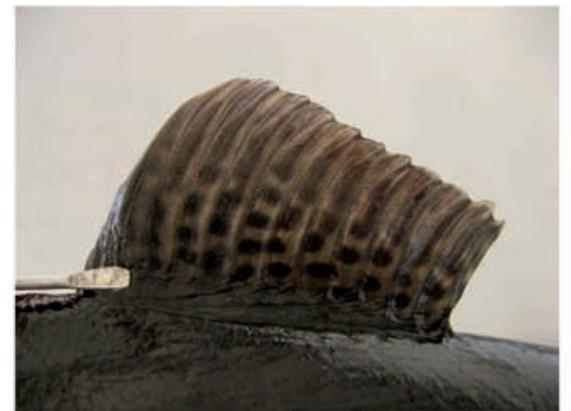

A, level 0

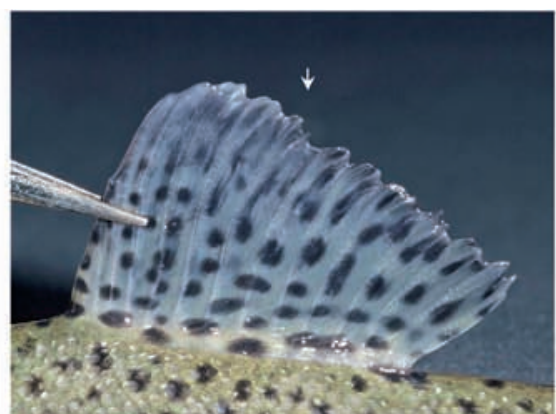

B, level 1

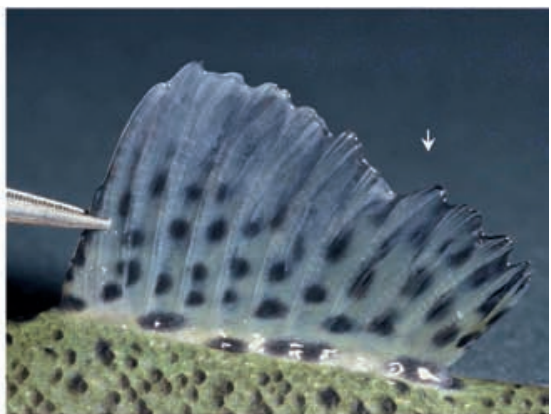

C, level 2

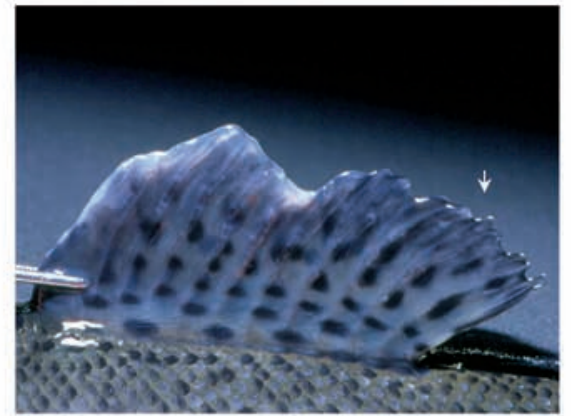

D, level 3

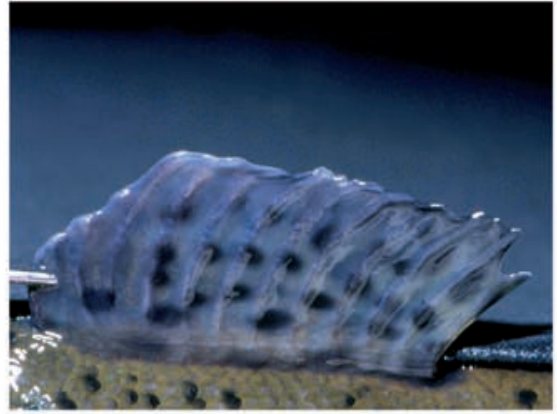

E, level 4

Fig. 4. Typical erosion levels of dorsal fin of $O$. mykiss . A, level 0; B, level 1; C, level 2, D level 3; E, level 4. The arrow indicates the rank of last intact ray; the slides are not at the same scale.

Dorsal fin length is estimated by sliding a scale along fin insertion to body until the highest length is observed (Fig. 1B).

Fin profile is characterized using 5 levels (Table 1). For practical reasons, the dorsal fin rays are not coded in the same direction than in pectoral fins. They are coded from back to front as reported in Fig. 2B (R1, the first posterior ray) and each degree of erosion refers to the rank of the last intact ray.

\section{Discussion}

This study provides a reference method to rapidly assess fin erosion in rainbow trout $(100-500 \mathrm{~g})$ with reference both to length and profile deterioration of the dorsal and pectoral fins using the highest fin length and the precise rank of intact or eroded rays to limit subjective bias or reference to a perfect fin. The method is easy to use for any operator with experience but requires little training and, it could be improved using digital processing to assess fin area. In comparison with other methods reviewed by Latremouille (2003) or recently proposed, it allows any operator to quantify in a reliable way small amounts of abrasion of two selected fins that are indicative of a change in fish welfare status prior to any deterioration of health condition. It is a direct method using anaesthetized fish to quantify within less than one minute the erosion of two fins (and not all rayed fins) without killing the fish. It does not need any special equipment and may be easily used to quantify fin erosion and possibly general fish welfare. Severe fin damage and other concomitant fish injuries are mainly indicative of bad health condition of fish and act as fish quality indicator, as a result recent methods assess both fish quality and fish welfare (St-Hilaire et al. 2006; Hoyle et al. 2007).

Fin length that may be expressed as relative fin indexes of specific fin or all fins are more easy to determine than erosion levels but it is less informative to assess welfare than fin profile. Fin relative length is dependent on fish species or strain, fish age or stage of development and fins are differently affected by culture conditions (water management, feeding, tank design) as shown in Salmonids (Bosakowski and Wagner 1994; Barrows and Lellis 1999; Pelis and McCormick 2003; St-Hilaire et al. 2006). Whereas it is relevant to control fish origin or the quality of juveniles at the time of release (Goede and Barton 1990; Latremouille 2006). By recording the maximum length of selected fins as described in this study observer bias is limited and, the standardized procedure proposed represents an improvement of Kindschi 1987's method.

The 5 erosion levels proposed gives an instantaneous picture of fin profile deterioration of pectoral and dorsal that can be used in farms, experiments and in the wild to evaluate fish welfare. The main difficulty is that there is not yet objective information on what is acceptable in terms of welfare (Ellis et al. 2002; Turnbull et al. 2005; Huntingford et al. 2006; Ashley 2007).

Fin abrasion is primarily caused by physical contact between fish or with rough surface and the changes with time in fin profile follow the same process at least in 100-500 g fish: first damages are observed at the same fin end and are followed by a progressive decrease in fin surface and length as described in this study. When there are con-specific attacks, often caused by inappropriate feeding procedures specially at high stocking 
densities, central portion of the dorsal and pectoral fins may be accidentally damaged while the extremities are intact. The occurrence of slits in the two selected fins seems mainly related to inadequate handling. As a first step it is suggested to specify if fins are whole or divided (major slits) and if bite marks, inflamed or haemorrhagic lesions are present using the qualitative clinical descriptors described in Hoyle et al. (2007). Fin examination may also provide information on recent life story of fish, following a damage fins may regenerate but never recover the initial appearance (Latremouille 2003; Hoyle et al. 2007). For example, ray deformations are indicative of bad welfare status at a certain period of life (poor management), and the presence of regenerating signs on fin tissues are indicative of recovery from necrosis (secondary infections may follow severe fin damages).

Further experiments are required to test the suitability of the two descriptors of fin erosion, fin length and profile of the dorsal and pectoral fins to analyze fin erosion causes under laboratory, farm or field conditions in comparison with other welfare indicators. The applicability of the method to rapidly pinpoint fin erosion alteration specially in large rainbow trout and other species and so to anticipate future changes in fish health condition compromising the economic value of fish should also be tested.

Acknowledgements. This study was part of a national contract (01/2 210488 , DGAL-INRA-IFREMER). The authors are grateful to the staff of Sizun and specially to L. Labbé for fish supply, to colleagues from IFREMER Brest, J. L. Gaignon, M. H. Omnes, B. Petton, L. Quéméner, A. Sévère for their help and advice and to A. Keay for help in improving English.

\section{References}

Arndt R.E., Routledge M.D., Wagner E.J., Mellethin R.F., 2002, The use of Aquamasts $($ to enhance growth and improve fin condition among raceway cultured rainbow trout Oncorhynchus mykiss (Walbaum). Aquac. Res. 33, 359-367.

Ashley P.J., 2007, Fish welfare: current issues in aquaculture. Appl. Anim. Behav. Sci. 104, 199-235.
Barrows F.T., Lellis W.A., 1999, The effect of dietary protein and lipid source on dorsal fin erosion in rainbow trout, Oncorhynchus mykiss. Aquaculture 180, 167-175.

Bosakowski T., Wagner E.J., 1994, Assessment of fin erosion by comparison of the relative fin length in hatchery and wild brown trout in Utah. Can. J. Fish. Aquat. Sci. 51, 636-641.

Bosakowski T., Wagner E.J., 1995, Experimental use of cobble substrates in concrete raceways for improving fin condition of cutthroat (Oncorhynchus clarki) and rainbow trout (O. mykiss). Aquaculture 130, 159-165.

Danner G.R., Boucher D.P., 2005, Relative pectoral fin length distinguishes between brook trout and landlocked Atlantic salmon fry. Northeast. Nat. 12, 503-508.

Ellis T., North, B.P., Scott A.P., Bromage N.R., Porter M.J., Gadd D., 2002, The relationships between stocking density and welfare in farmed rainbow trout. J. Fish Biol. 61, 493-531.

Geode R.W., Barton B.A., 1990, Organismic indices and an autopsybased assessment as indicators of health and condition of fish. Am. Fish. Soc. Symp. 8, 93-108.

Hoyle I., Oidtmann B., Ellis T., Turnbull J., North B., Nikolaidis J, Knowles T.G., 2007, A validated macroscopic key to assess fin damage in farmed rainbow trout (Oncorhynchus mykiss). Aquaculture (in press).

Huntingford F.A., Adams C.E., Braithwaite V.A., Kadri S., Pottinger T.G., Sandoe P., Turnbull J.F., 2006, Current issues in fish welfare. J. Fish Biol. 68, 332-372.

Kindschi G.A., 1987, Method for quantifying degree of fin erosion. Prog. Fish Cult. 49, 314-315.

Latremouille D.N., 2003, Fin erosion in aquaculture and natural environments. Rev. Fish. Sci. 11, 315-335.

Pelis R.M., McCormick S.D., 2003, Fin development in stream- and hatchery-reared Atlantic Salmon. Aquaculture 220, 525-536.

St Hilaire S., Ellis T., Cooke A., North B.P., Turnbull J.F., Knowles T., Kestin S., 2006, Fin erosion on rainbow trout on commercial trout farms in the United Kingdom. Vet. Record 159, 446-451.

Turnbull J.F., Bell A., Adams C.E., Bron J., Huntingford F.A., 2005, Stocking density and welfare of cage farmed Atlantic salmon: application of a multivariate analysis. Aquaculture 243, 121-132.

Zimmerman M.S., Krueger C.C., Eshenroder R.L., 2006, Phenotypic diversity of lake trout in great slave lake: differences in morphology, buoyancy, and habitat depth. Trans. Am. Fish. Soc. 135, 1056-1067. 